\title{
Approximate Bounds for Limited Backhaul Uplink Multicell Processing with Single-User Compression
}

\author{
Yuhan Zhou and Wei Yu \\ Department of Electrical and Computer Engineering \\ University of Toronto, Toronto, Ontario M5S 3G4, Canada \\ Emails: $\{$ yzhou,weiyu $\}$ comm.utoronto.ca
}

\begin{abstract}
This paper studies single-user compression for the uplink multi-cell processing (MCP) model where multiple basestations are connected to a central processor via noiseless backhaul links with a sum capacity constraint. The proposed scheme is based on the virtual multiple-access channel (V-MAC) architecture, where the base-stations quantize the received signals and send the quantized bits to the central processor, and at the decoder side the central processor first recovers the quantized signals then decodes the user messages in a successive manner. This paper focuses on the use of single-user compression in the V-MAC architecture, which is significantly simpler to implement than Wyner-Ziv compression. The main result of this paper is that single-user compression can achieve the sum capacity of the MCP model to within a constant gap under two specific scenarios. First, we define a diagonally dominant channel criterion and show the constant-gap result for the case when the covariance matrix of the received signals across the base-stations is $\kappa$-strictly diagonally dominant for some constant $\kappa>1$. Second, we show that the constant-gap result holds for a special class of Wyner channel models in the weak interference regime.
\end{abstract}

\section{INTRODUCTION}

Joint multi-cell processing (MCP) is a novel network architecture, in which the base-stations (BSs) can share information such as the transmitted and received signals and the channel state information with a centralized processor (CP) through high-capacity backhaul links. Under the MCP architecture, joint transmission in the downlink and joint reception in the uplink can be performed to effectively mitigate inter-cell interference. The MCP architecture has the potential to significantly improve the overall performance of wireless networks [1], [2].

This paper studies an uplink MCP architecture, known as the virtual multiple-access channel model (V-MAC) [3], in which the BSs act as relay terminals by first quantizing the received signals, then forwarding quantized bits to the $\mathrm{CP}$. In the decoding procedure, the $\mathrm{CP}$ first decodes the quantized signals, then decodes the user messages successively based on the quantized signals. The design of quantization schemes in such an architecture is crucial. This paper investigates the approximate optimality of single-user compression for the MCP model under a sum backhaul capacity constraint. Our contributions are two-fold: First, we show that single-user compression achieves the sum capacity of the MCP model to within a constant gap when the channel satisfies a diagonally dominant channel criterion. Second, we show that the constant gap-to-optimality result still holds for the Wyner soft-handoff model in the weak interference regime.
This paper is an extension of [3], where Wyner-Ziv compression is employed to quantize the signals received at the BSs. Since the received signals at different BSs are statistically correlated, Wyner-Ziv compression achieves higher coding efficiency than single-user compression. In fact, it has been shown in [3] that Wyner-Ziv compression can achieve the sum capacity of the MCP model to within a constant gap under a backhaul sum capacity constraint. However, WynerZiv compression is complex to implement in practice. In addition, it requires each BS to have global channel state information. For this reason, this paper chooses to replace Wyner-Ziv compression by single-user compression, and aims to show that single-user compression retains the constant-gap optimality in sum capacity under certain scenarios.

Achievability schemes for uplink MCP model under the individual backhaul capacity constraint have been studied extensively in the literature [4], [5], [6], [7], [8]. However, most existing schemes require joint decoding of the transmitted signals and the quantized signals, which is prohibitively complex to implement; the resulting rate region itself is also complex to evaluate. The main point of the current paper is that under a sum backhaul constraint, and roughly speaking for the channels with weakly correlated received signals, such complexity can be avoided by single-user compression and successive decoding without losing the approximate optimality in the sum capacity.

\section{Channel Model}

Fig. 1 illustrates the network topology considered in this paper, where $L$ mobile users communicate with a $\mathrm{CP}$ via $L$ BSs. The BSs are connected to the CP through noiseless backhaul links under a sum capacity constraint $C$. The users and the BSs are each equipped with a single antenna. BSs do not perform local decoding; all the user messages have to be decoded at the CP.

Defining $h_{i j}$ as the real channel gain between the $j$ th user and the $i$ th $\mathrm{BS}$, and let $X_{i} \sim \mathcal{N}\left(0, P_{i}\right)$ be the transmitted signal of the $i$ th user, then the $i$ th $\mathrm{BS}$ receives

$$
Y_{i}=\sum_{j=1}^{L} h_{i j} X_{j}+Z_{i} \quad \text { for } \quad i=1,2, \ldots, L,
$$

where the additive noise $Z_{i} \sim \mathcal{N}\left(0, \sigma^{2}\right)$ is independent and identically distributed (i.i.d.) over time. In this paper, 


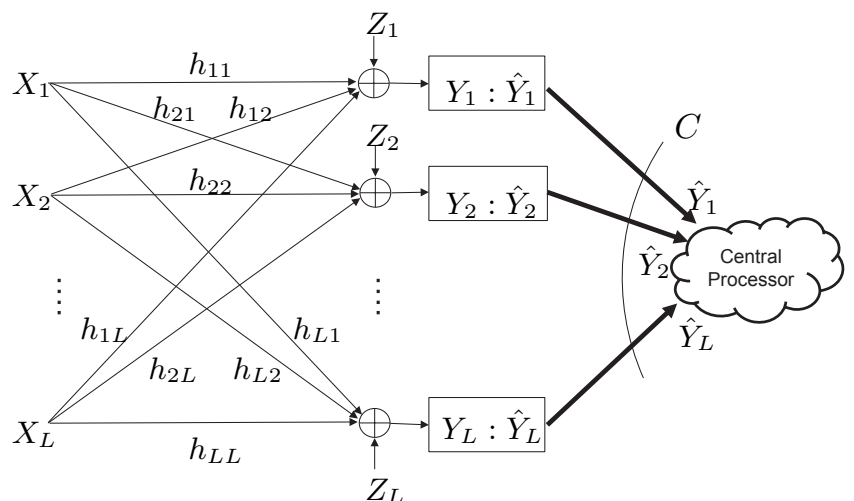

Fig. 1. Uplink multicell joint processing via a central processor

we assume that the average transmit powers $P_{i}$ 's are fixed. The received signal-to-noise ratio (SNR) at the $i$ th $\mathrm{BS}$ and interference-to-noise ratio (INR) from the $j$ th user to the $i$ th BS are defined as follows:

$$
\operatorname{SNR}_{i}=\frac{h_{i i}^{2} P_{i}}{\sigma^{2}}, \operatorname{INR}_{i, j}=\frac{h_{i j}^{2} P_{j}}{\sigma^{2}}, \text { for } i, j=1,2, \ldots, L .
$$

The notations used in this paper are as follows. Let $\mathbf{X}=$ $\left[X_{1}, X_{2}, \ldots, X_{L}\right]^{T}$ denote the transmitted signal vector. Let $\mathbf{Y}=\left[Y_{1}, Y_{2}, \ldots, Y_{L}\right]^{T}$ denote the received signal vector. Let $\widehat{\mathbf{Y}}=\left[\hat{Y}_{1}, \hat{Y}_{2}, \ldots, \hat{Y}_{L}\right]^{T}$ represent the quantized signal vector at the BSs. Let $q_{i}=\mathbb{E}\left(\hat{Y}_{i}-Y_{i}\right)^{2}$ be the average squarederror distortion between $Y_{i}$ and $\hat{Y}_{i}$. The distortion level $q_{i}$ is also referred to as the quantization noise level in this paper. We use $\mathbf{H}$ to denote the $L \times L$ channel matrix with entries $h_{i j}$, and $K_{X}=\mathbb{E}\left[\mathbf{X X}^{T}\right]$ to denote the covariance matrix of the transmitted signal vector. If $S \subseteq\{1,2, \ldots, L\}$, then $\mathbf{X}(S)$ is the subset of $\mathbf{X}$ with elements whose indices are the elements of $S$. Suppose that $K$ is a matrix, $\operatorname{diag}(K)$ denotes the diagonal matrix formed by the diagonal entries of $K$.

\section{V-MAC SCHEME WITH SINGLE-USER COMPRESSION}

\section{A. Achievable Rate Region}

This paper proposes a scheme in which each BS quantizes the received signals using single-user compression. By singleuser compression, we mean that the compression process does not take advantage of the statistical correlations between the received signals at different BSs. At the decoder side, the CP first decodes the quantized signals $\widehat{\mathbf{Y}}$, then decodes the user messages based on $\widehat{\mathbf{Y}}$. This coding scheme is referred to as the V-MAC-SU scheme in this paper, because the channel from $\mathbf{X}$ to $\widehat{\mathbf{Y}}$ forms a virtual multiple-access channel, and singleuser compression (SU) is used. This scheme differs from [3] only in the quantization part, where Wyner-Ziv compression is employed to fully explore the statistical correlation between the received signals across the BSs. As compared to Wyner-Ziv compression, single-user is more desirable in the wireless environment, since it only needs local channel state information at each BS. The following theorem characterizes the achievable rate region given by the V-MAC-SU scheme.
Theorem 1: For the uplink MCP model with $L$ BSs and sum backhaul capacity $C$ shown in Fig. 1, the following rate tuple $\left(R_{1}, R_{2}, \ldots, R_{L}\right)$ is achievable using the V-MAC-SU scheme:

$$
\sum_{i \in S} R_{i} \leq \frac{1}{2} \log \frac{\left|\mathbf{H}_{S} K_{X(S)} \mathbf{H}_{S}^{T}+\Lambda_{q}+\sigma^{2} I\right|}{\left|\Lambda_{q}+\sigma^{2} I\right|}
$$

subject to

$$
\frac{1}{2} \log \frac{\left|\operatorname{diag}\left(\mathbf{H} K_{X} \mathbf{H}^{T}\right)+\Lambda_{q}+\sigma^{2} I\right|}{\left|\Lambda_{q}\right|} \leq C
$$

where $K_{X(S)}=\mathbb{E}\left[\mathbf{X}(S) \mathbf{X}(S)^{T}\right]$ is the transmit signal covariance matrix, $\Lambda_{q}=\operatorname{diag}\left(q_{1}, q_{2}, \ldots, q_{L}\right)$ is the covariance matrix of the quantization noise, and $\mathbf{H}_{S}$ denotes the channel gain matrix from $\mathbf{X}(S)$ to $\mathbf{Y}$.

This theorem is a straightforward extension of [3, Theorem 1], where the rate expression (1) is given by the achievable sum rate $I(\mathbf{X}(S) ; \widehat{\mathbf{Y}})$ and the constraint (2) follows from the backhaul constraint $\sum_{i=1}^{L} I\left(Y_{i} ; \hat{Y}_{i}\right) \leq C$. The rate expression implicitly assumes the successive decoding of the quantization codewords first, then the transmitted signals.

\section{Main Results}

In this section, we demonstrate that the V-MAC-SU scheme is approximately optimal for sum capacity for the MCP model under certain scenarios. Intuitively, Wyner-Ziv compression is beneficial only when the received signals at the BSs are highly correlated. This paper makes this intuition precise by defining a diagonally dominant channel criterion, and shows that if the interference channel in the MCP model satisfies such a criterion, single-user compression can already achieve the sum capacity to within a constant gap. In addition, we show that single-user compression also gives a constant-gap result for the Wyner model in the weak interference regime.

\section{A. Approximate Sum Capacity for the Diagonally Dominant Channel}

For realistic wireless cellular networks, the received signal covariance matrix $\mathbb{E}\left[\mathbf{Y} \mathbf{Y}^{T}\right]=\mathbf{H} K_{X} \mathbf{H}^{T}+\sigma^{2} I$ across the BSs is often diagonally dominant. This is because in cellular communication the path losses from one mobile user to different BSs are distance dependent, and each mobile user is associated with the closest BS. In the following, we define a diagonally dominant criterion for matrices, and bound the determinant of a diagonally dominant matrix in term of its diagonal entries.

Definition 1: For a fixed constant $\kappa>1$, a $n \times n$ matrix $\Psi$ is said to be $\kappa$-strictly diagonally dominant if

$$
|\Psi(i, i)| \geq \kappa \sum_{j \neq i}^{n}|\Psi(i, j)| \text { for all } i=1, \ldots, L,
$$

where $\Psi(i, j)$ is the $(i, j)$-th entry of matrix $\Psi$.

Lemma 1: For fixed $\kappa>1$, suppose that a $n \times n$ matrix $\Psi$ is $\kappa$-strictly diagonally dominant, then

$$
|\Psi| \geq\left(1-\frac{1}{\kappa}\right)^{n} \prod_{i=1}^{n}|\Psi(i, i)| .
$$


Proof: The proof follows from the lower bound given in [9], which shows that if $\Psi$ is strictly diagonally dominant, i.e. $|\Psi(i, i)|>\sum_{j \neq i}^{n}|\Psi(i, j)|$ for $i=1, \ldots, n$, then the determinant of $\Psi$ can be bounded from below as follows,

$$
|\Psi| \geq \prod_{i=1}^{n}\left(|\Psi(i, i)|-\sum_{j \neq i}^{n}|\Psi(i, j)|\right) .
$$

Under the condition that $\Psi$ is $\kappa$-strictly diagonally dominant, i.e. $\sum_{j \neq i}^{n}|\Psi(i, j)| \leq \frac{|\Psi(i, i)|}{\kappa}$ we further bound $|\Psi|$ by

$$
\begin{aligned}
|\Psi| & \geq \prod_{i=1}^{n}\left(|\Psi(i, i)|-\frac{|\Psi(i, i)|}{\kappa}\right) \\
& =\left(1-\frac{1}{\kappa}\right)^{n} \prod_{i=1}^{n}|\Psi(i, i)|,
\end{aligned}
$$

which completes the proof.

Theorem 2: For the uplink MCP model with $L$ BSs and sum backhaul capacity $C$ shown in Fig. 1, if the received signal covariance matrix $\mathbf{H} K_{X} \mathbf{H}^{T}+\sigma^{2} I$ is $\kappa$-strictly diagonally dominant for a given constant $\kappa>1$, the V-MAC-SU scheme achieves the sum capacity to within $\frac{L}{2}\left(1+\log \frac{\kappa}{\kappa-1}\right)$ bits.

Proof: The proof uses the same technique as in [3]. We first choose the quantization noise levels $q_{i}=\alpha \sigma^{2}$, $i=1,2, \ldots, L$, where $\alpha>0$ is a constant depending on $C$, then compare the achievable rate of the V-MAC-SU scheme with the following cut-set like upper bound [5]

$$
\bar{C}=\min \left\{\frac{1}{2} \log \frac{\left|\mathbf{H} K_{X} \mathbf{H}^{T}+\sigma^{2} I\right|}{\left|\sigma^{2} I\right|}, C\right\} .
$$

We consider two different cases as follows: when $C \geq$ $\frac{1}{2} \log \frac{\left|\operatorname{diag}\left(\mathbf{H} K_{X} \mathbf{H}^{T}\right)+2 \sigma^{2} I\right|}{\left|\sigma^{2} I\right|}$, i.e. the sum backhaul capacity is large enough to support the choice of $q_{i}=\sigma^{2}$, we choose $\alpha=1$. In this case, the gap between $\bar{C}$ and $R_{\text {sum }}$ can be bounded by

$$
\begin{aligned}
\bar{C}-R_{\text {sum }} \leq & \frac{1}{2} \log \frac{\left|\mathbf{H} K_{X} \mathbf{H}^{T}+\sigma^{2} I\right|}{\left|\sigma^{2} I\right|} \\
& -\frac{1}{2} \log \frac{\left|\mathbf{H} K_{X} \mathbf{H}^{T}+2 \sigma^{2} I\right|}{\left|2 \sigma^{2} I\right|}<\frac{L}{2} .
\end{aligned}
$$

When $C<\frac{1}{2} \log \frac{\left|\operatorname{diag}\left(\mathbf{H} K_{X} \mathbf{H}^{T}\right)+2 \sigma^{2} I\right|}{\left|\sigma^{2} I\right|}$, we choose $\alpha$ so that $\sum_{i=1}^{L} I\left(Y_{i} ; \hat{Y}_{i}\right)=C$. First, notice that

$$
\sum_{i=1}^{L} I\left(Y_{i} ; \hat{Y}_{i}\right)=\frac{1}{2} \log \frac{\left|\operatorname{diag}\left(\mathbf{H} K_{X} \mathbf{H}^{T}\right)+(1+\alpha) \sigma^{2} I\right|}{\left|\alpha \sigma^{2} I\right|}
$$

is a monotonically decreasing function of $\alpha$. Since $C=$ $\sum_{i=1}^{L} I\left(Y_{i} ; \hat{Y}_{i}\right)<\frac{1}{2} \log \frac{\left|\operatorname{diag}\left(\mathbf{H} K_{X} \mathbf{H}^{T}\right)+2 \sigma^{2} I\right|}{\left|\sigma^{2} I\right|}$, we have $\alpha>1$. Now, we use $C=\sum_{i=1}^{L} I\left(Y_{i} ; \hat{Y}_{i}\right)$ as an upper bound. Let $\Omega=\mathbf{H} K_{X} \mathbf{H}^{T}+(1+\alpha) \sigma^{2} I$ and note that $\Omega(i, i) \geq 0$. The

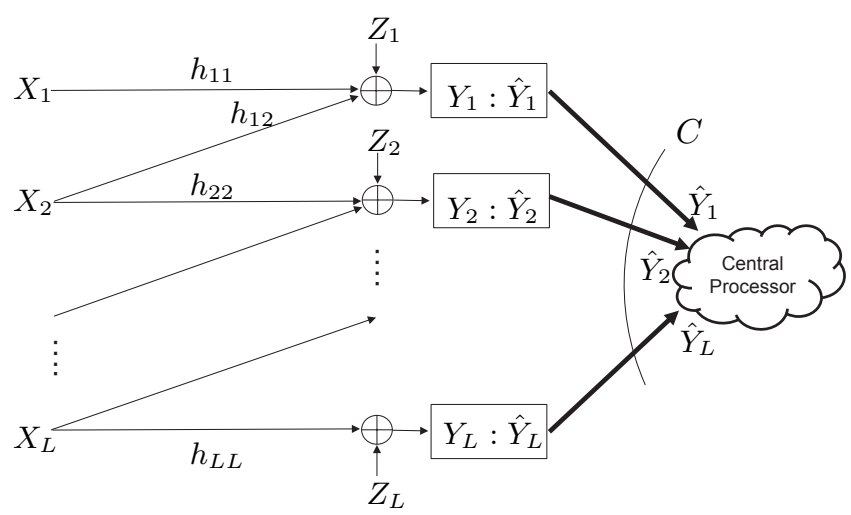

Fig. 2. Uplink multi-cell processing under Wyner soft-handoff model

gap between $\bar{C}$ and $R_{\text {sum }}$ is bounded by

$$
\begin{aligned}
\bar{C}-R_{\text {sum }} \leq & \sum_{i=1}^{L} I\left(Y_{i} ; \hat{Y}_{i}\right)-I(\mathbf{X} ; \widehat{\mathbf{Y}}) \\
= & \frac{1}{2} \log \frac{\left|\operatorname{diag}\left(\mathbf{H} K_{X} \mathbf{H}^{T}\right)+(1+\alpha) \sigma^{2} I\right|}{\left|\alpha \sigma^{2} I\right|} \\
& -\frac{1}{2} \log \frac{\left|\mathbf{H} K_{X} \mathbf{H}^{T}+(1+\alpha) \sigma^{2} I\right|}{\left|(1+\alpha) \sigma^{2} I\right|} \\
= & \frac{1}{2} \log \left[\left(1+\frac{1}{\alpha}\right)^{L} \frac{\prod_{i=1}^{L} \Omega(i, i)}{|\Omega|}\right] .
\end{aligned}
$$

Since matrix $\mathbf{H} K_{X} \mathbf{H}^{T}+\sigma^{2} I$ is $\kappa$-strictly diagonally dominant, $\Omega$ is also $\kappa$-strictly diagonally dominant. Following the result of Lemma 1, we further bound the gap as follows,

$$
\begin{aligned}
\bar{C}-R_{\text {sum }} & \leq \frac{L}{2} \log \left(1+\frac{1}{\alpha}\right)+\sum_{i=1}^{L} \frac{1}{2} \log \frac{\kappa}{\kappa-1} \\
& <\frac{L}{2}\left(1+\log \frac{\kappa}{\kappa-1}\right),
\end{aligned}
$$

where the last inequality follows from the fact that $\alpha>1$.

Combining the two cases, we see that the gap to sum capacity for the V-MAC-SU scheme with uniform quantization noise levels across the BSs is always less than $\frac{L}{2}\left(1+\log \frac{\kappa}{\kappa-1}\right)$.

Theorem 2 demonstrates that when $C$ is sufficiently large, setting the quantization noise levels to be at the background noise level results in at most $1 / 2$ bits gap per dimension per user to the cut-set bound. It is not hard to further verify that, in this case, the V-MAC-SU scheme is actually approximately optimal for the entire capacity region of the MCP model. When $C$ is small, an extra condition on $\mathbf{H} K_{X} \mathbf{H}^{T}+\sigma^{2} I$, which requires the received signals across the BSs to be weakly correlated, is needed to preserve the constant-gap-to-optimality result.

\section{B. Approximate Sum Capacity for Wyner model}

In the following, we demonstrate the approximate optimality of the V-MAC-SU scheme in the case where the interference channel is partially connected. More specifically, we consider 
the Wyner soft-handoff model [10], where each transmitterreceiver pair interferes only one of its neighboring receivers and gets interfered by only one of its other neighboring transmitters, as shown in Fig. 2. Despite its simplicity, the Wyner model captures the essential structure of cellular networks, and it is widely adopted in theoretical analysis. The constant-gapto-optimality result of the V-MAC-SU scheme for the Wyner model is stated as follows

Theorem 3: For the uplink multi-cell processing Wyner model with sum backhaul capacity $C$ shown in Fig. 2, in the weak interference regime where $\operatorname{INR}_{i, i+1} \leq \mathrm{SNR}_{i}, i=$ $1,2, \ldots, L-1$, the V-MAC-SU scheme achieves a sum rate that is within $L$ bits to the sum capacity.

Proof: Under the Wyner model, the channel can be written as

$$
\begin{aligned}
& Y_{i}=h_{i i} X_{i}+h_{i, i+1} X_{i+1}+Z_{i}, \quad \text { for } \quad i=1, \ldots, L-1 \\
& Y_{L}=h_{L L} X_{L}+Z_{L}
\end{aligned}
$$

Let quantization noise levels $q_{i}=\alpha \sigma^{2}$, for $i=1, \ldots, L$, we have the sum rate

$$
\begin{aligned}
R_{\text {sum }} & =\sum_{i=1}^{L} I\left(X_{i} ; \hat{Y}_{1}, \ldots, \hat{Y}_{L} \mid X_{i+1}^{L}\right) \geq \sum_{i=1}^{L} I\left(X_{i} ; \hat{Y}_{i} \mid X_{i+1}^{L}\right) \\
& =\sum_{i=1}^{L} I\left(X_{i} ; \hat{Y}_{i} \mid X_{i+1}\right)=\sum_{i=1}^{L} \frac{1}{2} \log \frac{\mathrm{SNR}_{i}+1+\alpha}{1+\alpha}
\end{aligned}
$$

and $C \geq \sum_{i=1}^{L} I\left(Y_{i} ; \hat{Y}_{i}\right)=\sum_{i=1}^{L} \frac{1}{2} \log \frac{\mathrm{SNR}_{i}+\mathrm{INR}_{i, i+1}+1+\alpha}{\alpha}$.

We choose $\alpha$ using the same strategy as in Theorem 2 and also use the same upper bound (6). When the sum backhaul capacity $C$ is large enough to support the quantization noise levels to be at the background noise level, we choose $\alpha=1$, i.e., if $C \geq \sum_{i=1}^{L} \frac{1}{2} \log \left(2+\operatorname{SNR}_{i}+\operatorname{INR}_{i, i+1}\right)$, set $q_{i}=\sigma^{2}$. Similar to what is shown in the proof of Theorem 2, the gap between the upper bound $I(\mathbf{X} ; \mathbf{Y})$ and the achievable sum rate $I(\mathbf{X} ; \widehat{\mathbf{Y}})$ in this case is bounded by $\frac{L}{2}$.

When $C<\sum_{i=1}^{L} \frac{1}{2} \log \left(2+\operatorname{SNR}_{i}+\operatorname{INR}_{i, i+1}\right)$, we choose $q_{i}=\alpha \sigma^{2}$, where $\alpha$ is chosen to make $C=$ $\sum_{i=1}^{L} \frac{1}{2} \log \frac{\mathrm{SNR}_{i}+\mathrm{INR}_{i, i+1}+1+\alpha}{\alpha}$. Again, based on the fact that

$$
\sum_{i=1}^{L} I\left(Y_{i} ; \hat{Y}_{i}\right)=\sum_{i=1}^{L} \frac{1}{2} \log \frac{\operatorname{SNR}_{i}+\operatorname{INR}_{i, i+1}+1+\alpha}{\alpha}
$$

is a monotonically decreasing function of $\alpha$, we have $\alpha>1$. Now the gap between $\bar{C}$ and $R_{\text {sum }}$ is bounded by

$$
\begin{aligned}
\bar{C}-R_{\text {sum }} \leq & \sum_{i=1}^{L} \frac{1}{2} \log \frac{\operatorname{SNR}_{i}+\operatorname{INR}_{i, i+1}+1+\alpha}{\alpha} \\
& -\sum_{i=1}^{L} \frac{1}{2} \log \frac{\operatorname{SNR}_{i}+1+\alpha}{1+\alpha} \\
< & \frac{L}{2}+\sum_{i=1}^{L} \frac{1}{2} \log \left(1+\frac{1}{\alpha}\right)<L
\end{aligned}
$$

where the second inequality bases on the fact that $\operatorname{INR}_{i, i+1} \leq$ $\mathrm{SNR}_{i}$, and the last inequality follows from the fact that $\alpha>1$.
Combining the two cases, we conclude that the gap to the sum capacity for the V-MAC-SU scheme with uniform quantization noise levels is always less than $L$.

It is worthy to note that the conclusion of Theorem 3 does not follow from Theorem 2. For instance, consider a Wyner soft-handoff model with two BSs, where $h_{11}=h_{22}=1$, $h_{12}=\gamma, P_{1}=P_{2}=1$, and $\sigma^{2}$ is close to 0 . If $\gamma^{2} \leq 1$, then the weak interference condition in Theorem 3, i.e., $\mathrm{INR}_{i, i+1} \leq \mathrm{SNR}_{i}$, is satisfied, thus the V-MAC-SU scheme achieves a sum rate which is at most 2 bits away from the sum capacity regardless of the value of SNR. However, in this model,

$$
\mathbf{H} K_{X} \mathbf{H}^{T}+\sigma^{2} I \approx\left[\begin{array}{cc}
1+\gamma^{2} & \gamma \\
\gamma & 1
\end{array}\right] .
$$

To satisfy the condition that $\mathbf{H} K_{X} \mathbf{H}^{T}+\sigma^{2} I$ is $\kappa$-strictly diagonally dominant, we have to choose a constant $\kappa=1$. For this choice of $\kappa$, we cannot get a bounded gap to the sum capacity based on Theorem 2 .

\section{CONClusion}

This paper studies an uplink MCP model where the cell sites are linked to a $\mathrm{CP}$ via noiseless backhaul links with a finite sum capacity. We propose a V-MAC-SU scheme, where the received signals are first quantized at the BSs by singleuser compression, then transmitted to the $\mathrm{CP}$. It is shown that the V-MAC-SU scheme achieves the sum capacity of the MCP model to within a constant gap, when either the received signal covariance matrix $\mathbf{H} K_{X} \mathbf{H}^{T}+\sigma^{2} I$ is $\kappa$-strictly diagonally dominant for some constant $\kappa>1$ or the channel can be modeled under the Wyner soft-handoff model in the weak interference regime.

\section{REFERENCES}

[1] D. Gesbert, S. Hanly, H. Huang, S. Shamai, O. Simeone, and W. Yu, "Multi-cell MIMO cooperative networks: A new look at interference," IEEE J. Sel. Areas Commun., vol. 28, no. 9, pp. 1380-1408, Dec. 2010.

[2] P. Marsch and G. Fettweis, "Uplink CoMP under a constrained backhaul and imperfect channel knowledge," IEEE Trans. Wireless Commun., vol. 10, no. 6, pp. 1730-1742, June 2011.

[3] Y. Zhou, W. Yu, and D. Toumpakaris, "Uplink multi-cell processing: Approximate sum capacity under a sum backhaul constraint," Submitted to IEEE Information Theory Workshop (ITW), 2013. [Online]. Available: http://arxiv.org/abs/1304.7509

[4] A. Sanderovich, S. Shamai, Y. Steinberg, and G. Kramer, "Communication via decentralized processing," IEEE Trans. Inf. Theory, vol. 54, no. 7, pp. 3008-3023, July 2008.

[5] A. Sanderovich, O. Somekh, H. V. Poor, and S. Shamai, "Uplink macro diversity of limited backhaul cellular network," IEEE Trans. Inf. Theory, vol. 55, no. 8, pp. 3457-3478, Aug. 2009.

[6] A. Sanderovich, S. Shamai, and Y. Steinberg, "Distributed MIMO receiver - Achievable rates and upper bounds," IEEE Trans. Inf. Theory, vol. 55, no. 10, pp. 4419-4438, Oct. 2009.

[7] A. Avestimehr, S. Diggavi, and D. Tse, "Wireless network information flow: A deterministic approach," IEEE Trans. Inf. Theory, vol. 57, no. 4, pp. 1872-1905, Apr. 2011.

[8] S. H. Lim, Y.-H. Kim, A. El Gamal, and S.-Y. Chung, "Noisy network coding," IEEE Trans. Inf. Theory, vol. 57, no. 5, pp. 3132-3152, May 2011.

[9] A. M. Ostrowski, "Note on bounds for determinants with dominant principal diagonal," Proc. Amer. Math. Soc., vol. 3, no. 1, pp. 26-30, 1952.

[10] A. Wyner, "Shannon-theoretic approach to a Gaussian cellular multipleaccess channel," IEEE Trans. Inf. Theory, vol. 40, no. 6, pp. 1713-1727, Nov. 1994. 